Research Paper

\title{
External Radiation versus Internal Radiation for Patients with Advanced Unresectable HCC -A SEER Based Study
}

\author{
Xuan Wang\#, Mo Chen", Ran Wei", Zheng Wang ${ }^{\circledR}$ \\ Department of General Surgery, Huashan Hospital \& Cancer Metastasis Institute, Fudan University, Shanghai, China \\ \#these authors contributed equally to this work. \\ $\triangle$ Corresponding author: Zheng Wang, MD, Department of General Surgery, Huashan Hospital \& Cancer Metastasis Institute, Fudan university, 12 Urumqi \\ Road (M), Shanghai 200040, P.R. China. Tel: \& Fax: +86-21-5288-7172; E-mail: wilsonwangzheng@163.com \\ (C) Ivyspring International Publisher. This is an open access article distributed under the terms of the Creative Commons Attribution (CC BY-NC) license \\ (https://creativecommons.org/licenses/by-nc/4.0/). See http://ivyspring.com/terms for full terms and conditions.
}

Received: 2018.08.03; Accepted: 2018.12.05; Published: 2019.01.29

\begin{abstract}
Hepatocellular carcinoma ( $\mathrm{HCC})$, 2nd most lethal cancer globally, is the major type of primary liver malignancies currently resulting in about 800,000 deaths globally per annum. Surgical resection remains the only curative treatment to HCC, which does not fit for many patients diagnosed with advanced HCC. Radiation therapy has been gradually concerned as an optional treatment for patients with advanced unresectable HCC. This study aimed to compare external radiation (beam radiation) and internal radiation as a single radiation therapy to advanced HCC patients (TNA stage III and IV according to the derived AJCC Stage, 6th edition) stratified by other risk factors, based on the data collected from the Surveillance, Epidemiology and End Results (SEER) national database. In this cohort study, we mainly clarified the following three points: 1 . Choices of radiation therapy for advanced HCC patients vary in demographic and clinical factors. Among these, TNM classification is the key factor. 2. Internal radiation provides a better prognosis in both OS and CSS. 3. Patients in stage IV could benefit from internal radiation preferentially, while for patients in stage III, internal radiation therapy makes no difference compared with external radiation.
\end{abstract}

Key words: Hepatocellular carcinoma, radiation therapy, SEER

\section{Introduction}

Primary Malignancy in liver has become one of the most common and lethal malignant tumors worldwide, presenting a severe threat to the life quality of patients (1). Hepatocellular carcinoma (HCC), the second most lethal cancer globally (2), is the major type of primary liver malignancy $(85-90 \%)$ (3), currently resulting in about 800,000 deaths globally per annum $(4,5)$, with even increasing incidence and related-mortality in multiple regions and nations especially like the USA and East Asia countries (5).

Optimal treatment selections for HCC can be varied based on a diverse spectrum of severity in patients (6). Surgical treatments including surgical hepatic resection and liver transplantation have been regarded as potentially curative treatments for HCC, providing a far better long-term relapse-free survival rates $(40 \%)$ and five-year survival rates $(90 \%)$ than other alternatives in carefully selected patients $(7,8)$. Speaking of the surgical standard, the existing BCLC (Barcelona Clinic Liver Cancer) staging system and Milano criteria have been questioned in one way or another $(9,10)$. In order to get a better prognosis for the patients, sometimes can even be the majority, failing to meet the indications of surgical treatments, alternative options like radiations, ablation and chemotherapy need considering.

Radiation therapy has been gradually concerned as an optional treatment for patients with advanced unresectable HCC (11). Fortunately, HCC is a radiosensitive tumor located in a radiosensitive organ, suggesting the possible effectiveness of radiotherapy (12). Yet solid clinical evidence has been absent to justify the effectiveness and safety of 
radiotherapy. According to the data documented in the SEER database, conventional radiotherapy of HCC can be classified into basically two types, the external and internal radiotherapy. The former mainly refers to the beam radiation therapy, parallel radiation beam from external origin directly kills the tumor lesion. While the latter refers to implantation of radioactive particles, like radioactive isotopes adjacent to the primary malignancy, providing continuous and accurate radiation $(13,14)$. Both of them have been served in clinical practice for treating advanced HCC, but with vague boundaries and unspecific target populations. Missing data and evidence fail to confirm a personalized radiotherapy guideline and evaluation pattern for prognosis. The SEER database only provides information on radiation therapy methods, without mentioning data relating to aims (palliative or curative), doses and

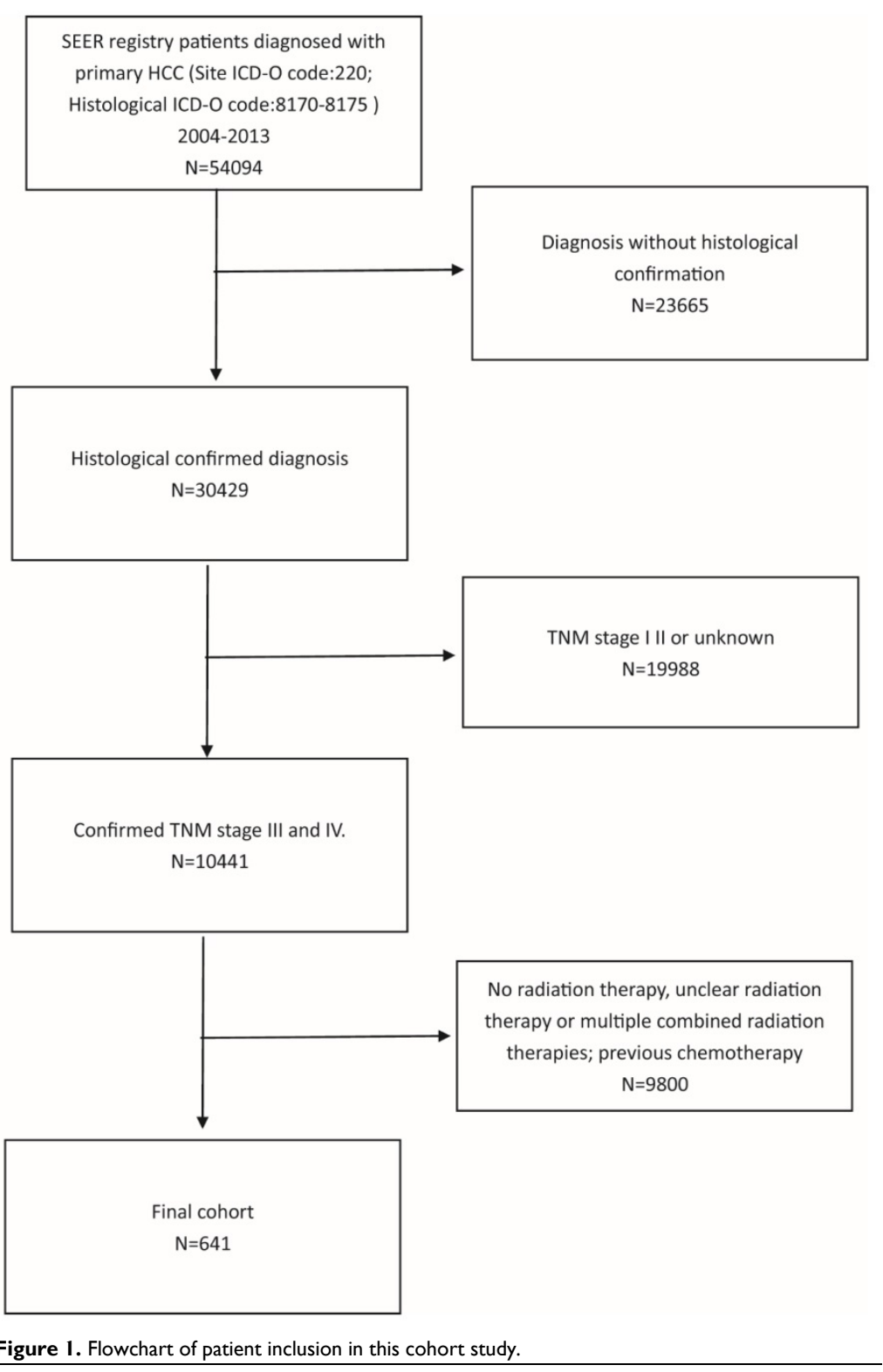

schemes. Therefore we decided to focus on comparison between internal and external radiation therapies.

This study aimed to compare external radiation (beam radiation) and internal radiation as a single radiation therapy to advanced HCC patients (TNA stage III and IV according to the derived AJCC Stage, $6^{\text {th }}$ edition) stratified by other risk factors. We examined OS and CSS of these radiation therapies and tried to come up with optimal radiation schemes for HCC patients in multiple states.

\section{Materials and methods}

We applied data collected from the Surveillance, Epidemiology and End Results (SEER) national database to perform a retrospective cohort study. Identification of patients with confirmed HCC diagnosis was done by utilizing site ICD-0-code 220 and histological ICD-0-code 8170-8175. Patients diagnosed with solid histological evidence were further selected. Moreover, we specially enrolled patients with TNM stage III and IV (according to the derived AJCC Stage, $6^{\text {th }}$ edition). Among these chosen patients, we finally identified in total 641 patients with a single and certain radiation therapy (beam radiation or internal radiation) meeting all the criteria above (Figure 1). Approval was obtained from the Shanghai Huashan hospital Review Board and a data use agreement was completed for this project. Data was abstracted using the SEER Stat Software, version 8.4, National Cancer Institute, Washington DC.

Characteristics analysis including gender, marriage, age (stratified in groups), race, tumor grade, size (stratified in groups), lymph invasion and TNM classification. The reason why we did not include metastasis is that whether the tumor has distally metastasized or not determines the TNM classification to be IV or III. In these circumstances, metastasis status would be one and the same thing as TNM classification. Cohort stratification of patients was based on the types of radiation therapy: beam radiation and internal radiation. Beam radiation is labeled by radiation code in SEER database as "beam radiation", while the latter includes both of the labels "radioisotope" and "radioactive implants". As mentioned above, in order to get a better comparison, we further stratified age and size into 
subgroups separately with the assistance of X-tile software: $\leq 50,50-69$ and $\geq 70$ years; $<3.0,3.0-5.0$ and $>5.0 \mathrm{~cm}$.

Table 1. Characteristics of patients with advanced liver cancer in SEER database.

\begin{tabular}{|c|c|c|c|c|c|c|}
\hline Parameter & Characteristic & $\begin{array}{l}\text { Number } \\
\text { of } \\
\text { patients }\end{array}$ & \multirow[t]{2}{*}{$\begin{array}{l}\text { Beam } \\
\text { radiation } \\
\mathrm{N}(\%)\end{array}$} & $\begin{array}{l}\begin{array}{l}\text { Internal } \\
\text { radiation }\end{array} \\
\mathrm{N}(\%)\end{array}$ & $X^{2}$ & $\begin{array}{l}\mathrm{P} \\
\text { value }\end{array}$ \\
\hline \multirow[t]{3}{*}{ Gender } & & & & & 0.746 & 0.388 \\
\hline & Male & 542 & $416(76.8)$ & $126(23.2)$ & & \\
\hline & Female & 99 & $72(72.7)$ & $27(27.3)$ & & \\
\hline \multirow[t]{7}{*}{ Marriage } & & & & & 24.531 & $<0.001$ \\
\hline & Married & 348 & $242(69.5)$ & $106(30.5)$ & & \\
\hline & Divorced & 84 & $74(88.1)$ & $10(11.9)$ & & \\
\hline & Seperated & 7 & $6(85.7)$ & $1(14.3)$ & & \\
\hline & $\begin{array}{l}\text { Single (never } \\
\text { married) }\end{array}$ & 124 & $103(83.1)$ & $21(16.9)$ & & \\
\hline & Widowed & 55 & 47 (85.5) & $8(14.5)$ & & \\
\hline & Unknown & 22 & $16(72.7)$ & $6(27.3)$ & & \\
\hline \multirow[t]{4}{*}{ Age } & & & & & 2.095 & 0.351 \\
\hline & $\leq 50$ & 49 & 39 (79.6) & $10(20.4)$ & & \\
\hline & $51-69$ & 367 & $285(77.7)$ & $82(22.3)$ & & \\
\hline & $\geq 70$ & 225 & $164(72.9)$ & $61(27.1)$ & & \\
\hline \multirow[t]{4}{*}{ Race } & & & & & 0.345 & 0.841 \\
\hline & White & 454 & $344(75.8)$ & $110(24.2)$ & & \\
\hline & Black & 107 & $81(75.7)$ & $26(24.3)$ & & \\
\hline & ${ }^{*}$ Others & 80 & $63(78.8)$ & $17(21.3)$ & & \\
\hline \multirow[t]{6}{*}{ Grade } & & & & & 58.232 & $<0.001$ \\
\hline & I-Well differentiated & 82 & $44(53.7)$ & $38(46.3)$ & & \\
\hline & $\begin{array}{l}\text { II-Moderately } \\
\text { differentiated }\end{array}$ & 99 & 57 (57.6) & $42(42.4)$ & & \\
\hline & $\begin{array}{l}\text { III-Poorly } \\
\text { differentiated }\end{array}$ & 78 & $64(82.1)$ & $14(17.9)$ & & \\
\hline & IV-Undifferentiated & 2 & $2(100)$ & $0(0)$ & & \\
\hline & Unknown & 380 & $321(84.5)$ & $59(15.5)$ & & \\
\hline \multirow[t]{7}{*}{$\begin{array}{l}\text { Tumor } \\
\text { stage }\end{array}$} & & & & & 138.285 & $<0.001$ \\
\hline & T0 & 9 & $9(100)$ & $0(0.0)$ & & \\
\hline & $\mathrm{T} 1$ & 125 & $115(92.0)$ & $10(8.0)$ & & \\
\hline & $\mathrm{T} 2$ & 67 & $60(89.6)$ & $7(10.4)$ & & \\
\hline & $\mathrm{T} 3$ & 259 & $136(52.4)$ & $123(47.5)$ & & \\
\hline & $\mathrm{T} 4$ & 42 & 34 (96.4) & $8(19.0)$ & & \\
\hline & $\mathrm{TX}$ & 139 & $134(96.4)$ & $5(3.6)$ & & \\
\hline \multirow[t]{4}{*}{ Size $(\mathrm{cm})$} & & & & & 59.184 & $<0.001$ \\
\hline & $<3.0$ & 60 & 55 (91.7) & $5(8.3)$ & & \\
\hline & $3.0-5.0$ & 70 & $59(84.3)$ & 11 (15.7) & & \\
\hline & $>5.0$ & 317 & $200(63.1)$ & $117(36.9)$ & & \\
\hline \multirow{4}{*}{$\begin{array}{l}\text { Lymph } \\
\text { node } \\
\text { invasion }\end{array}$} & & & & & 34.879 & $<0.001$ \\
\hline & N0 & 433 & $305(70.4)$ & $128(29.6)$ & & \\
\hline & N1 & 83 & 63 (75.9) & $20(24.1)$ & & \\
\hline & $\mathrm{NX}$ & 125 & $120(96.0)$ & $5(4.0)$ & & \\
\hline \multirow[t]{3}{*}{ TNM stage } & & & & & 310.846 & $<0.001$ \\
\hline & III & 184 & $54(29.3)$ & $130(70.7)$ & & \\
\hline & IV & 457 & $434(95.0)$ & $23(5.0)$ & & \\
\hline
\end{tabular}

*Others include American Indian/AK Native, Asian/Pacific Islander.

\section{Results}

\section{Patients characteristic}

Among the total 641 patients selected based on the criteria, comparisons in demographic and clinical baseline data between the two different radiation groups were done (Table 1). Demographically, we found that married patients seemed more likely to accept internal radiation than those who were unmarried. This could be related to the higher cost of the internal radiation, as marital status may indicate, to a certain degree, the economic status. From the clinical aspect, patients with better differentiated, lager and restricted local invasion (T3) HCC are more likely to receive internal radiation. Moreover, TNM stage is another decisive factor. Most patients (70.7\%) with stage III accepted internal radiation therapy, while $95 \%$ IV patients chose external radiation.

\section{Survival analysis}

As shown in Table 2, we primarily conducted univariate analysis via Kaplan-Miere method to explore the varied significance that each demographic or clinical factor has on patients' overall survival (OS) and cancer-specific survival (CSS). In univariate analysis, to our surprise, age turned out to be one influential factor to CSS and it demonstrated a trend that elder patients got even longer lifespans. Consistent with our conventional knowledge, almost all the pathological factors (grade, T, N, M, tumor size and tumor stage) were proved to influence OS and CSS with statistical significance. However, as the survival curves (Figure 2, Figure 3) indicated, separated influence of some stratified factors was not straightforward enough, like grade, $\mathrm{T}$, tumor size and $\mathrm{N}$ stage. But integrated TNM classification and radiotherapy groups showed significant survival difference. Clearly noted from the table and figures, advanced stage suggested a worse prognosis in both OS and CSS. Similarly, internal radiotherapy provided a much better prognosis in OS and CSS than external ones.

We further applied Cox survival analysis to verify whether the potential risk factors can be viewed as independent prognosis indicators (Table 2, Figure 4 and Figure 5). Among all the potential demographic and clinical risk factors mentioned above, three factors ( $\mathrm{T}$, TNM stage and radiation therapy) were identified as independent prognosis indicators for OS and four (age, T, TNM stage and radiation therapy) for CSS. To be specific, except for T3 (HR: 0.935(0.696-1.256)) and T4 (HR: 1.093(0.849-1.409)), a more advanced $\mathrm{T}$ stage suggested a worse prognosis of patients. This is similar to TNM stage that IV may indicate much worse overall survival than III. These two factors behaved analogically in CSS. As for age, patients older than 70 years old surprisingly owned longer cancer-specific lifespans. This may be contradicted to our previous recognition. The most inspiring result was that for patients accepting internal radiation therapy properly, both overall survival and cancer-specific survival prognosis are much better (for OS 1.488(1.159-1.912), for CSS 1.626(1.214-2.176)). Finally, Cox analysis revealed that 
patients of differed races had differed clinical outcomes in CSS statistically.

\section{Radiation therapy selection}

Considering there exists such diverse distributions in the two radiation therapies for patients of differed TNM classifications, we further grouped the patients based on their TNM stages (III and IV) to examine how differed choices of radiation could influence the patients' prognosis. Previous Cox analysis revealed that internal radiation can provide a better result in both overall survival and cancer-specific survival. However, in patients of stage III, external and internal radiation made no significant differences in OS and CSS. On the contrary, even though most stage IV patients chose external radiation, internal radiation was actually beneficial to patients in lifespan, both OS and CSS (Figure 6).

\section{Discussion}

Owing to its rapid exacerbation and surgical unresectability, advanced HCC has always been a severe challenge to the welfare of patients (15). Nowadays, beyond surgical interventions, integrated therapies provide a fresh perspective for advanced malignancy treatment (16). Radiation therapy, which used to be a palliative option for HCC treatment, now was proved to be effective for the selected advanced HCC patients $(17,18)$.

Table 2. Univariate and multivariate survival analyses for evaluating OS and CSS of advanced liver cancer patients in SEER database.

\begin{tabular}{|c|c|c|c|c|c|c|c|c|c|c|}
\hline Parameter & 6-month OS & Univariate analys & & Multivariate analys & & 6-month CSS & Univariate analysis & Multivo & riate analysis & \\
\hline & & Log rank $\chi^{2}$ test & $\mathrm{P}$ & $\mathrm{HR}(95 \% \mathrm{CI})$ & $\mathrm{P}$ & & Log rank $\chi 2$ test & $\mathrm{P}$ & HR $(95 \%$ CI) & $\mathrm{P}$ \\
\hline Sex & & $<0.001$ & 0.986 & & $\mathrm{NI}$ & & 0.429 & 0.513 & & NI \\
\hline Male & $39.1 \%$ & & & & & $46.7 \%$ & & & & \\
\hline Female & $35.2 \%$ & & & & & $47.3 \%$ & & & & \\
\hline Age & & 3.080 & 0.214 & & NI & & 11.927 & 0.003 & & 0.012 \\
\hline$<=50$ & $34.7 \%$ & & & & & $39.6 \%$ & & & Reference & \\
\hline $51-69$ & $36.0 \%$ & & & & & $42.9 \%$ & & & $1.372(0.942-1.999)$ & 0.099 \\
\hline$\geq 70$ & $43.5 \%$ & & & & & $54.9 \%$ & & & $1.366(1.108-1.684)$ & 0.003 \\
\hline Race & & 2.087 & 0.352 & & NI & & 1.916 & 0.384 & & 0.047 \\
\hline White & $37.4 \%$ & & & & & $45.0 \%$ & & & Reference & \\
\hline Black & $39.3 \%$ & & & & & $48.7 \%$ & & & $0.876(0.678-1.131)$ & \\
\hline *Others & $43.6 \%$ & & & & & $54.4 \%$ & & & $0.690(.510-0.933)$ & \\
\hline Marital status & & 7.807 & 0.253 & & NI & & 14.114 & 0.028 & & $\mathrm{NI}$ \\
\hline Married & $40.8 \%$ & & & & & $50.7 \%$ & & & & \\
\hline Divorced & $32.1 \%$ & & & & & $34.4 \%$ & & & & \\
\hline Separated & $42.9 \%$ & & & & & $42.9 \%$ & & & & \\
\hline Single & $33.1 \%$ & & & & & $40.4 \%$ & & & & \\
\hline Widowed & $38.2 \%$ & & & & & $48.1 \%$ & & & & \\
\hline Unknown & $50.0 \%$ & & & & & $52.9 \%$ & & & & \\
\hline Grade & & 10.685 & 0.030 & & $\mathrm{NI}$ & & 15.210 & 0.004 & & NI \\
\hline I & $47.6 \%$ & & & & & $58.2 \%$ & & & & \\
\hline II & $51.5 \%$ & & & & & $62,7 \%$ & & & & \\
\hline III & $33.3 \%$ & & & & & $39.9 \%$ & & & & \\
\hline IV & NI & & & & & NI & & & & \\
\hline Unknown & $34.2 \%$ & & & & & $41.3 \%$ & & & & \\
\hline Tumor stage & & 45.614 & $<0.001$ & & 0.014 & & 35.743 & $<0.001$ & & 0.040 \\
\hline T0 & $22.2 \%$ & & & Reference & & $25.9 \%$ & & & Reference & \\
\hline $\mathrm{T} 1$ & $36.8 \%$ & & & $1.380(0.702-2.712)$ & 0.350 & $46.9 \%$ & & & $1.107(0.447-2.742)$ & 0.826 \\
\hline $\mathrm{T} 2$ & $34.3 \%$ & & & $0.735(0.575-0.940)$ & 0.014 & $40.4 \%$ & & & $0.736(0.557-0.973)$ & 0.031 \\
\hline T3 & $49.0 \%$ & & & $0.935(0.696-1.256)$ & 0.655 & $55.9 \%$ & & & $1.025(0.740-1.419)$ & 0.883 \\
\hline $\mathrm{T} 4$ & $42.9 \%$ & & & 1.093 (0.849-1.409) & 0.490 & $48.1 \%$ & & & $1.133(0.853-1.504)$ & 0.389 \\
\hline Unknown & $22.3 \%$ & & & $0.717(0.497-1.036)$ & 0.077 & $31.6 \%$ & & & $0.704(0.456-1.086)$ & 0.112 \\
\hline Size $(\mathrm{cm})$ & & 20.731 & $<0.001$ & & $\mathrm{NI}$ & & 15.990 & 0.001 & & NI \\
\hline$\leq 3.0$ & $40.0 \%$ & & & & & $46.2 \%$ & & & & \\
\hline $3.1-4.9$ & $41.4 \%$ & & & & & $49.5 \%$ & & & & \\
\hline$>=5.0$ & $44.8 \%$ & & & & & $52.3 \%$ & & & & \\
\hline Unknown & $27.1 \%$ & & & & & $36.5 \%$ & & & & \\
\hline Lymph node invasion & & 16.475 & $<0.001$ & & NI & & 15.330 & $<0.001$ & & NI \\
\hline Negative & $43.2 \%$ & & & & & $51.3 \%$ & & & & \\
\hline Positive & $37.3 \%$ & & & & & $42.5 \%$ & & & & \\
\hline TNM stage & & 94.664 & $<0.001$ & & $<0.001$ & & 79.049 & $<0.001$ & & $<0.001$ \\
\hline III & $66.2 \%$ & & & Reference & & $71.3 \%$ & & & Reference & \\
\hline IV & $27.4 \%$ & & & $0.429(0.356-0.517)$ & $<0.001$ & $36.0 \%$ & & & $0.450(0.330-0.614)$ & $<0.001$ \\
\hline Radiation therapy & & 73.309 & $<0.001$ & & 0.001 & & 64.526 & $<0.001$ & & 0.001 \\
\hline Beam radiation & $29.1 \%$ & & & Reference & & $37.4 \%$ & & & Reference & \\
\hline Internal radiation & $68.6 \%$ & & & 1.488 (1.159-1.912) & 0.001 & $74.5 \%$ & & & $1.626(1.214-2.176)$ & \\
\hline
\end{tabular}



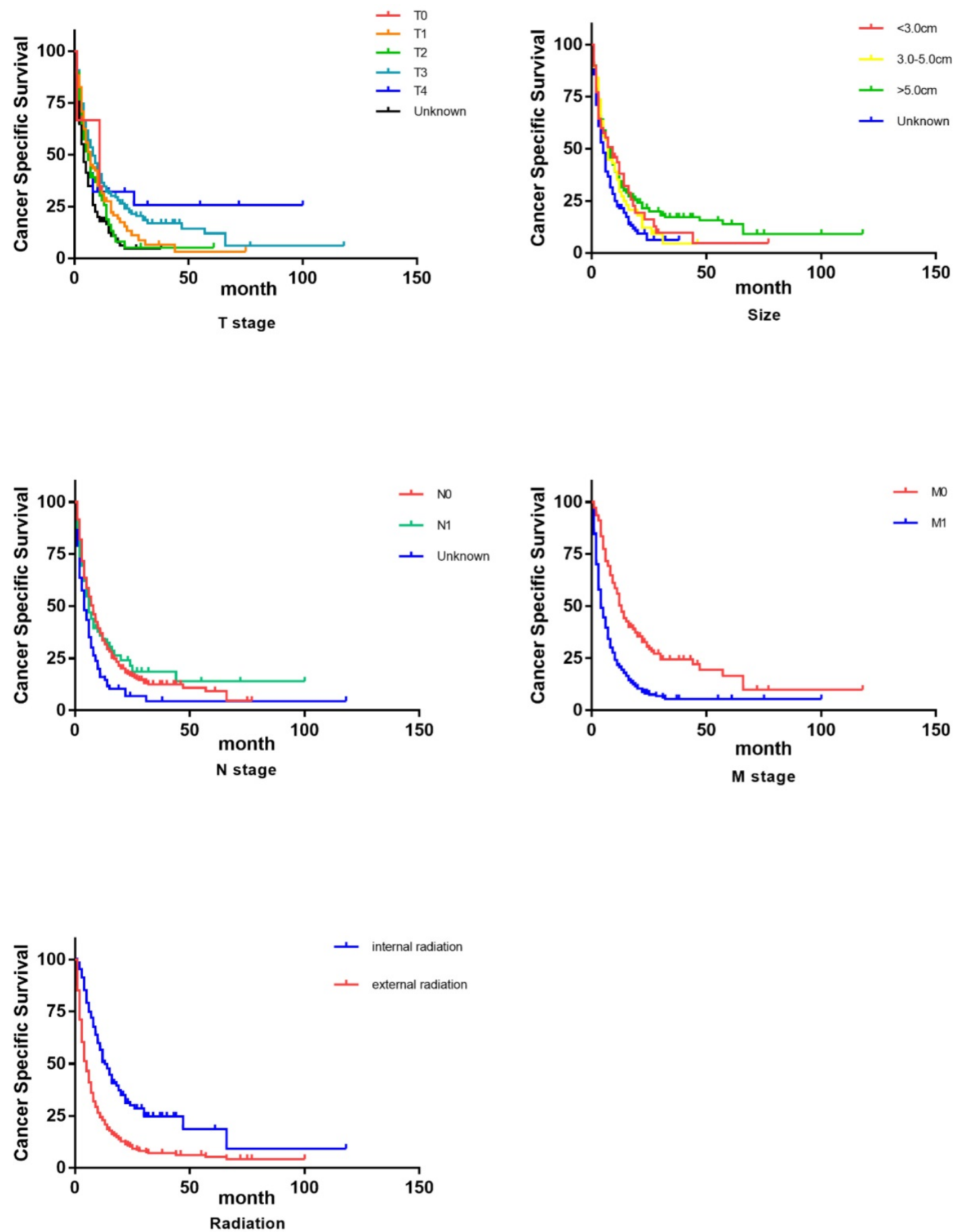

Figure 2. Survival curves on CSS of influential factors via Kaplan-Miere univariate analysis.

In this cohort study, we mainly clarified the following three points: Firstly, choices of radiation therapy for advanced HCC patients vary in demographic and clinical factors. Among these, TNM classification is the key factor. Secondly, internal radiation provides a better prognosis in both OS and CSS. Moreover, patients in stage IV could benefit from internal radiation preferentially, while for patients in stage III, internal radiation therapy makes no difference compared with external radiation.
The current guidelines have proposed the choice of radiotherapy as a conversion or an adjuvant therapy when primary HCC was diagnosed as surgically unresectable (19). However, selection of a single radiation protocol based on patients' circumstances still remains empirical. Various demographic and clinical factors can partially influence patients' selection on radiation therapy. In this study, we found that marital status can be one influential factor. Specifically, married patients are 
more likely to accept internal radiation therapy than those who live alone (divorced, separated and single as well as widowed). This may be correlated to social-economic influence because internal radiation cost more than external one while married person usually has a medical insurance.

From a pathological point of view, it is natural that tumor characteristics determine the selection of radiation. As concluded in this study, tumor stage,
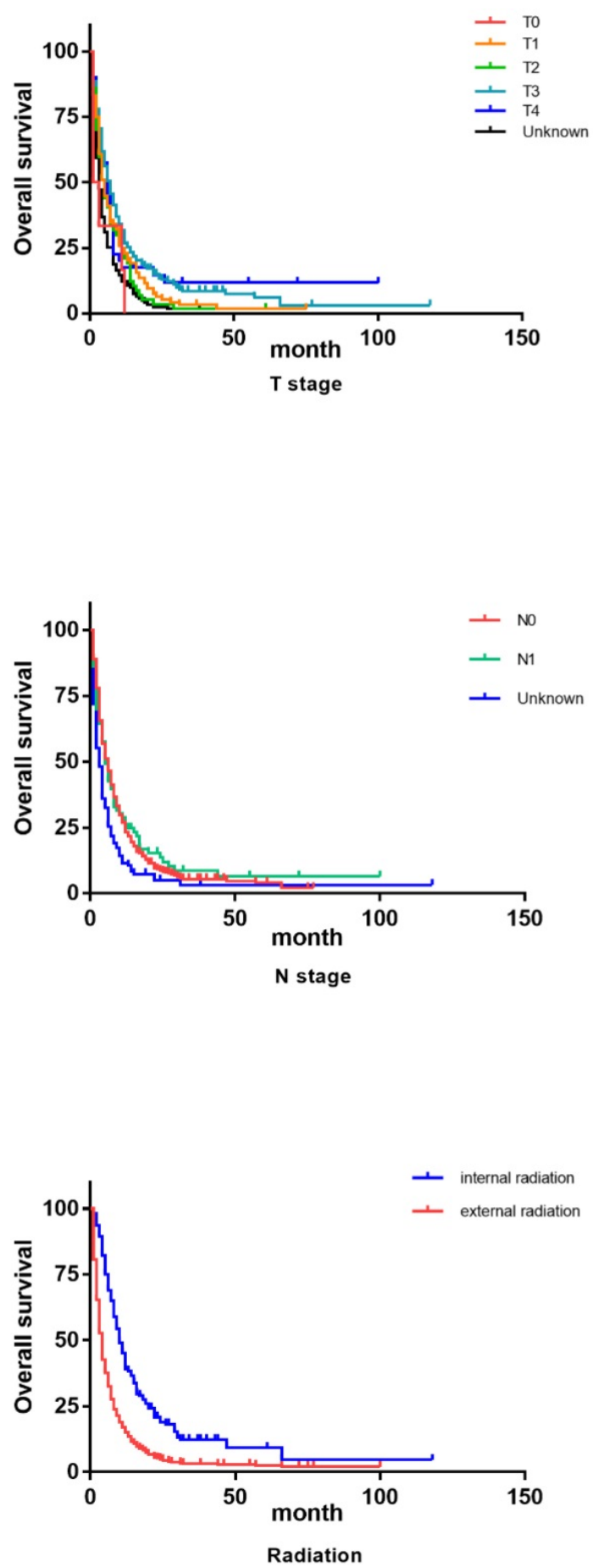

lymph node stage as well as tumor size are proved to be influential elements in radiation therapy selection. However, TNM stage would be the integration of the factors above, thus it can be a guideline for protocol choice of each patient. Although tumor differentiation grade was also a significant factor for radiation therapy according to our results. Nevertheless in clinical practice, grade information gained from non-surgical biopsy is not completely reliable.
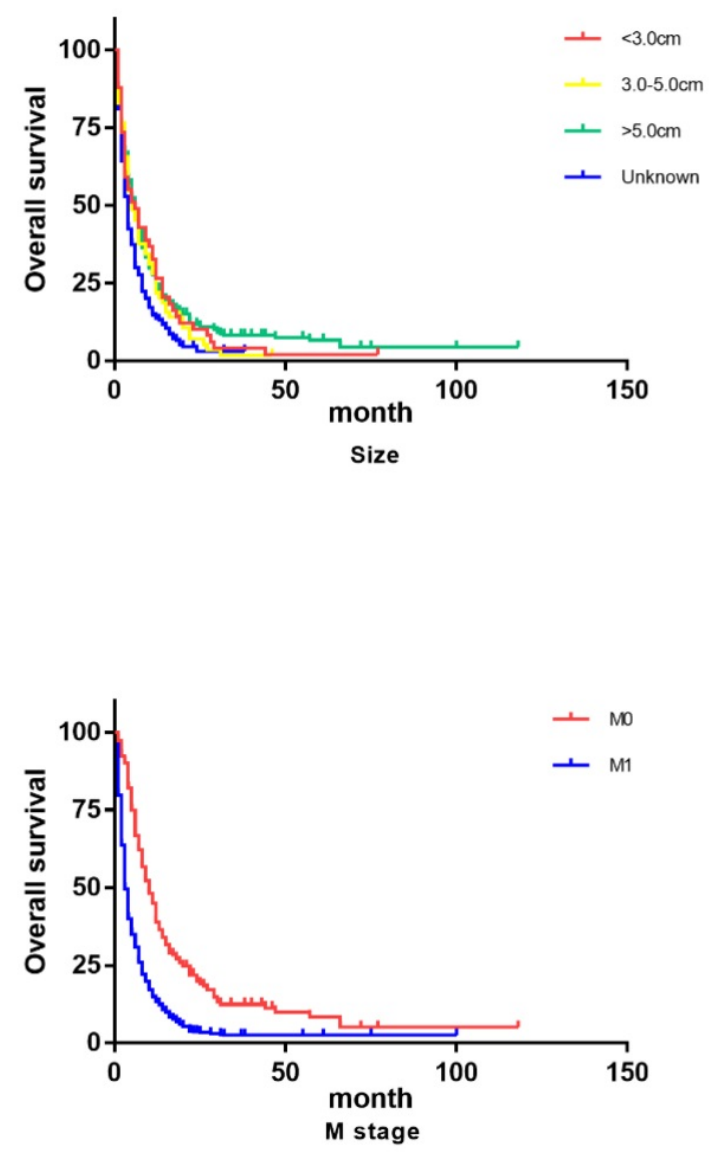

Figure 3. Survival curves on OS of influential factors via Kaplan-Miere univariate analysis 

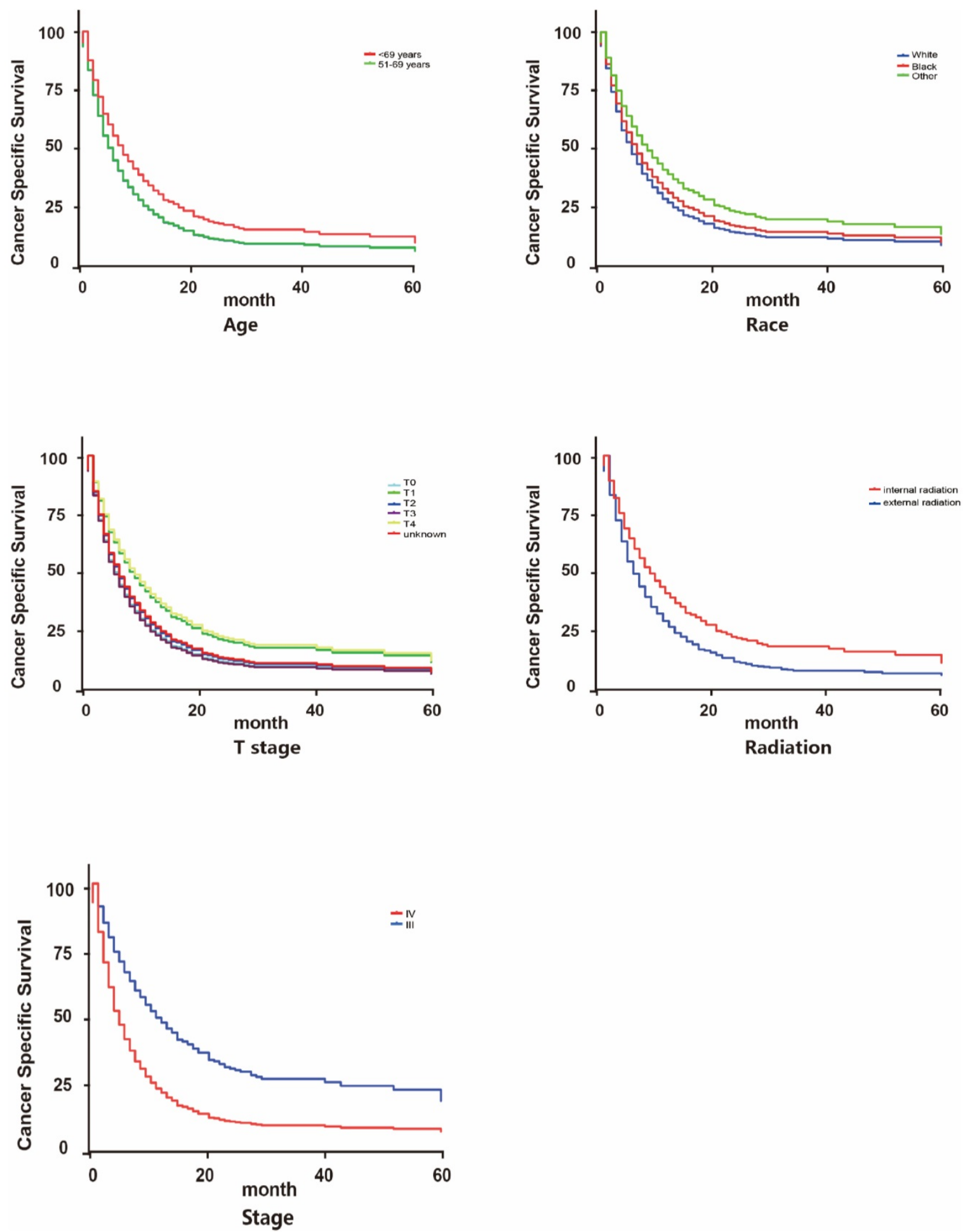

Figure 4. Survival curves on CSS of influential factors via Cox multivariate analysis

In univariate and multivariate survival analysis, we spotted that age was an independent indicator on HCC patients' cancer-specific survival. Elder patients (especially over 70s) seemed to have a longer cancer-specific survival. This may be explained by more frequent tumor screening for elder patients. Further explorations can be done to clarify that how age can influence the prognosis of advanced HCC patients. Besides, race can be suspected as an independent prognostic indicator for advanced patients among demographical factors. This actually is not the first time that studies revealed the impact of potential racial diversity on malignancy outcomes (20). 

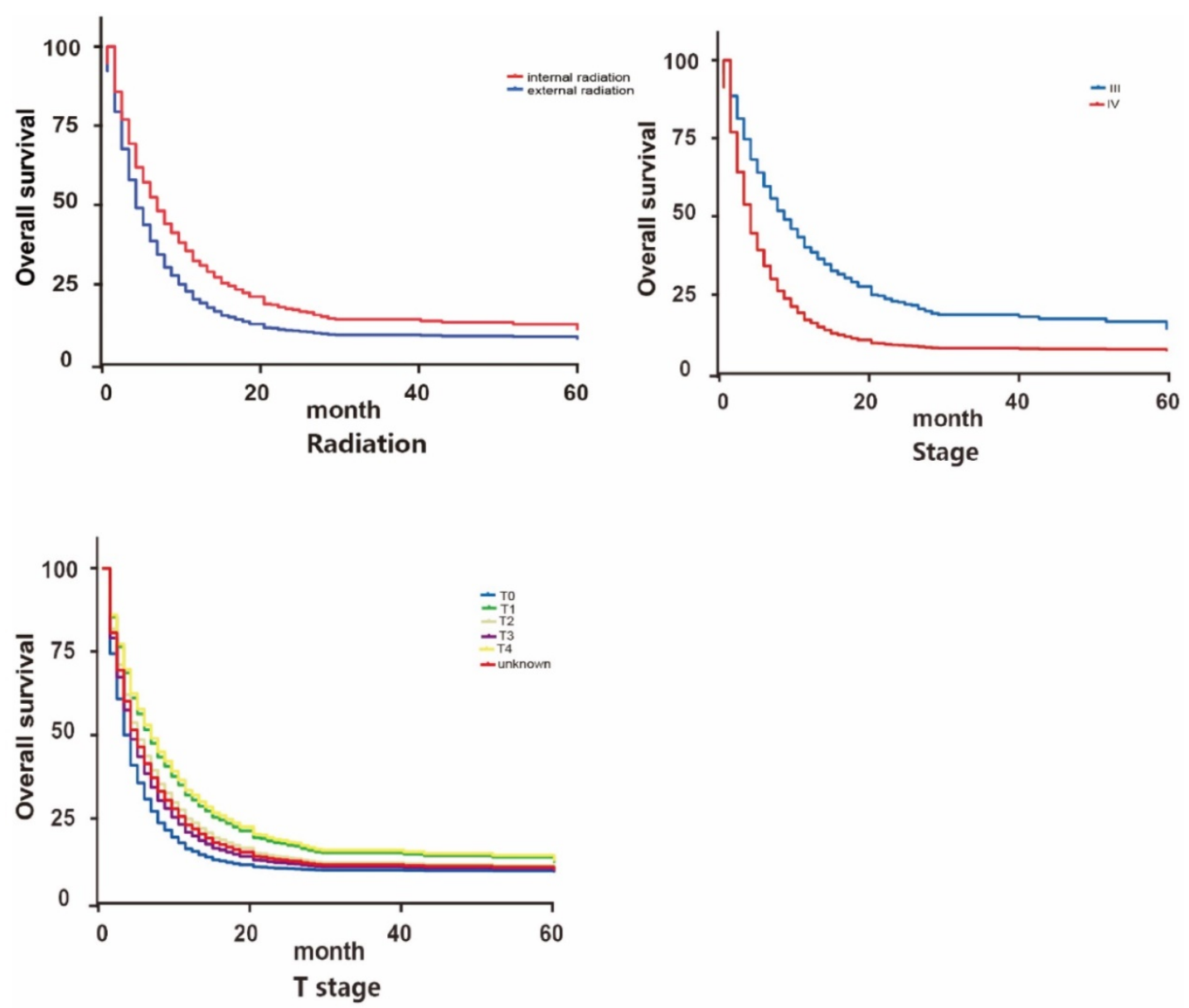

Figure 5. Survival curves on OS of influential factors via Cox multivariate analysis

The benefit of internal radiation varies in patients with differed TNM classifications. For patients with stage IV, internal radiation has a much better prognosis in both OS and CSS than external radiation. While for patients with stage III, there exists no significant variance between internal and external radiation. These discoveries endow this study with clinical significance. Previously, according to some empirical applications, patients with distal metastasis (stage IV) are more likely to be recommended to accept beam radiation. While for stage III patients, internal radiation can be preferred for its high efficiency $(18,21)$. These results we got could challenge our conventional routine and stimulate more innovations on current clinical practice.

As far as we are concerned, this SEER based study is among the pioneering work to investigate radiation protocol selection for advanced unresectable HCC patients. Still, limitations remain as a retrograde study. Initially, all the enrolled patients were diagnosed with HCC between 2004 and 2013. During more than one decade, radiation techniques keep involving to provide better treatments. This possibly resulted in bias while we calculate survival analysis for those who were diagnosed lately may accept improved radiation therapies for better prognosis. Also, owing to the documents of SEER database, the number of patients in different subgroup is not strictly matched. Moreover, though we classified the radiation therapies into mainly two types. It was actually not a very detailed classification. Owing to the missing information on aims (palliative or curative), schemes and specific doses of the radiation therapies from SEER database, our study was not able to further explore prognostic value of these detailed-classified radiation therapies. Last but not least, though we excluded interference by chemotherapy, it would be more convincing if we can stratified radiation therapy based on the order with surgery.

Conclusively, in this SEER based cohort study, we described the diverse distributions on radiation protocol selections for advanced unresectable HCC patients. More importantly, we explored that patients with TNM stage IV can benefit more from internal radiation while for stage III patients internal or external radiation makes no differences in long-term prognosis. These findings can be contradicted to 

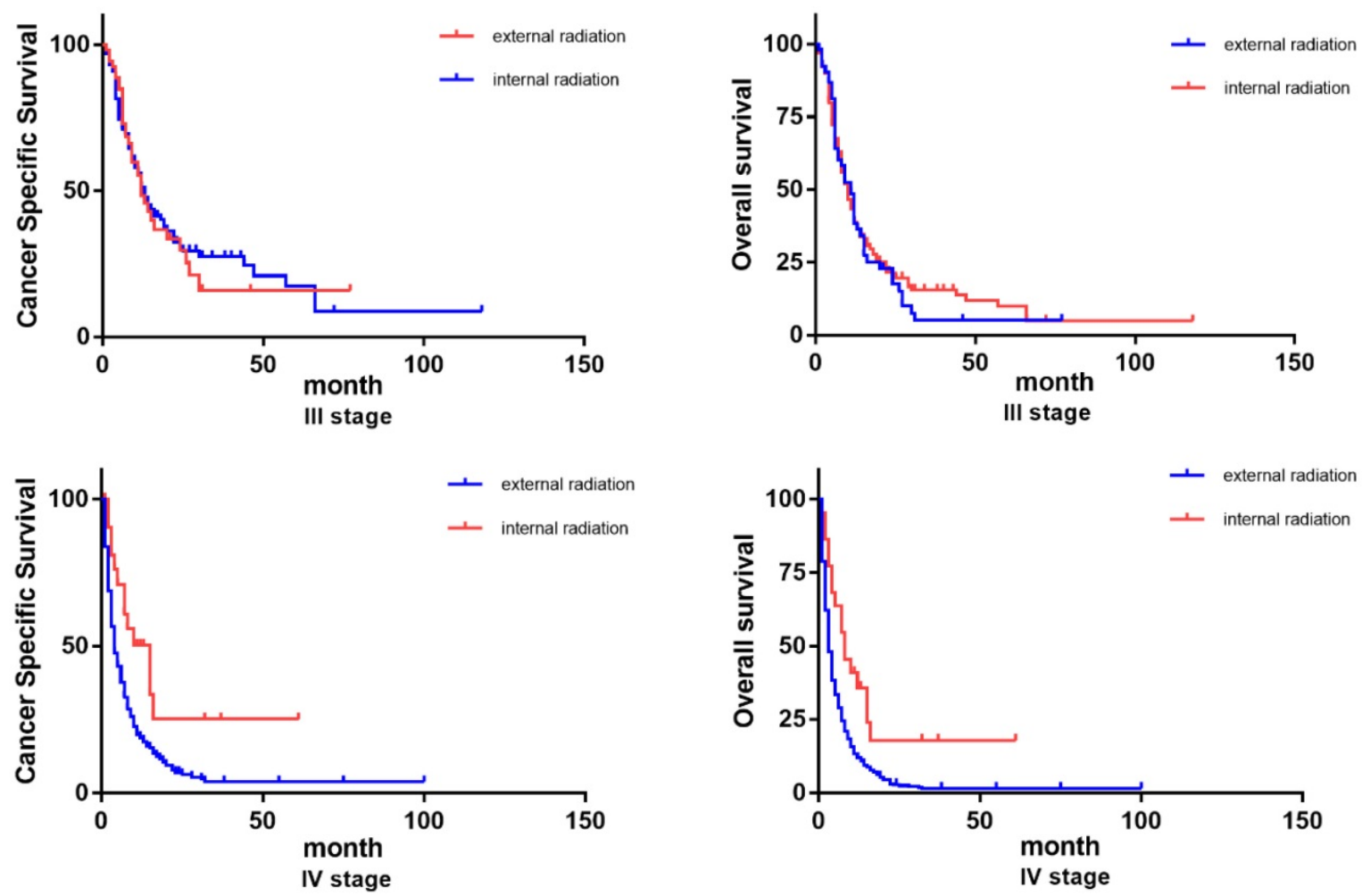

Figure 6. Survival curves on OS and CSS of patients with diverse TNM stages.

conventional thoughts. Hopefully, this study could provide a challenging insight and a new guide for future clinical practice.

\section{Acknowledgements}

This study was supported by Shanghai Sailing Program (No.17YF1401600), the National Natural Science Foundation of China (No. 81672820, No. 81472677 and No.81672365) and the National Key Project for Infectious Disease of China (No. 2017ZX10203207).

All of the authors have participated actively in this study, and agree to the content of the manuscript and their being listed as an author on the paper.

\section{Competing Interests}

The authors have declared that no competing interest exists.

\section{References}

1. La Vecchia C, Negri E. A review of epidemiological data on epilepsy, phenobarbital, and risk of liver cancer. Eur J Cancer Prev. 2014;23(1):1-7.

2. Toyoda H, Kumada T, Tada T, Mizuno K, Hiraoka A, Tsuji K, et al. Impact of Hepatocellular Carcinoma Etiology and Liver Function on the Benefit of Surveillance: a Novel Approach for the Adjustment of Lead-time Bias. Liver Int. 2018;38:2260-8

3. Mittal S, El-Serag HB. Epidemiology of hepatocellular carcinoma: consider the population. J Clin Gastroenterol. 2013;47 Suppl:S2-6.

4. Altekruse SF, Henley SJ, Cucinelli JE, McGlynn KA. Changing hepatocellular carcinoma incidence and liver cancer mortality rates in the United States. Am J Gastroenterol.2014;109(4):542-53.

5. Njei B, Rotman Y, Ditah I, Lim JK. Emerging trends in hepatocellular carcinoma incidence and mortality. Hepatology. 2015;61(1):191-9.
6. Mancuso A. Management of hepatocellular carcinoma: Enlightening the gray zones. World J Hepatol. 2013;5(6):302-10.

7. Mancuso A, Perricone G. Hepatocellular Carcinoma and Liver Transplantation: State of the Art. J Clin Transl Hepatol. 2014;2(3):176-81.

8. Cauchy F, Fuks D, Belghiti J. HCC: current surgical treatment concepts. Langenbecks Arch Surg. 2012;397(5):681-95.

9. Han K, Kim JH. Transarterial chemoembolization in hepatocellular carcinoma treatment: Barcelona clinic liver cancer staging system. World J Gastroenterol. 2015;21(36):10327-35.

10. Cauchy F, Soubrane O, Belghiti J. Liver resection for HCC: patient's selection and controversial scenarios. Best Pract Res Clin Gastroenterol. 2014;28(5):881-96.

11. Klein J, Dawson LA. Hepatocellular carcinoma radiation therapy: review of evidence and future opportunities. Int J Radiat Oncol Biol Phys. 2013;87(1):22-32.

12. Kim EH, Kim MS, Cho CK, Jung WG, Jeong YK, Jeong JH. Low and high linear energy transfer radiation sensitization of HCC cells by metformin. J Radiat Res. 2014;55(3):432-42.

13. Kalogeridi MA, Zygogianni A, Kyrgias G, Kouvaris J, Chatziioannou S, Kelekis N, et al. Role of radiotherapy in the management of hepatocellular carcinoma: A systematic review. World J Hepatol. 2015;7(1):101-12.

14. Malfertheiner P, Verslype C, Kolligs FT, Schutte K, Vandecaveye V, Paprottka $\mathrm{PM}$, et al. The effectiveness of selective internal radiation therapy in challenging cases of liver-predominant unresectable hepatocellular carcinoma. Future Oncol. 2014;10(15 Suppl):17-27.

15. Balogh J, Victor DR, Asham EH, Burroughs SG, Boktour M, Saharia A, et al. Hepatocellular carcinoma: a review. J Hepatocell Carcinoma. 2016:3:41-53.

16. Chen SW, Lin LC, Kuo YC, Liang JA, Kuo CC, Chiou JF. Phase 2 study of combined sorafenib and radiation therapy in patients with advanced hepatocellular carcinoma. Int J Radiat Oncol Biol Phys. 2014;88(5):1041-7.

17. Gallicchio R, Nardelli A, Mainenti P, Nappi A, Capacchione D, Simeon V, et al. Therapeutic Strategies in HCC: Radiation Modalities. Biomed Res Int. 2016;2016:1295329.

18. Lee IJ, Seong J. The optimal selection of radiotherapy treatment for hepatocellular carcinoma. Gut Liver. 2012;6(2):139-48.

19. Carlson RW, Larsen JK, McClure J, Fitzgerald CL, Venook AP, Benson AR, et al. International adaptations of NCCN Clinical Practice Guidelines in Oncology. J Natl Compr Canc Netw. 2014;12(5):643-8.

20. Ha J, Yan M, Aguilar M, Bhuket T, Tana MM, Liu B, et al. Race/ethnicity-specific disparities in cancer incidence, burden of disease, and overall survival among patients with hepatocellular carcinoma in the United States. Cancer-Am Cancer Soc. 2016;122(16):2512-23.

21. Yoon HI, Seong J. Multimodality treatment involving radiotherapy for advanced liver-confined hepatocellular carcinoma. Oncology-Basel. 2014;87 Suppl 1:90-8. 


\section{Author biography}

Dr. Xuan Wang is currently a M.D. in Cancer Metastasis Institute, Fudan University and the chief resident in the department of General Surgery, Huashan Hospital. His research currently is centered on the mechanism of early metastasis of hepatobiliary and GI malignancies.

Dr. Mo Chen is currently a M.D. in Cancer Metastasis Institute, Fudan University and resident in the department of General Surgery, Huashan Hospital. His research currently is centered on the mechanism of early metastasis of hepatobiliary and GI malignancies.

Dr. Ran Wei is currently a M.D. in Cancer Metastasis Institute, Fudan University and resident in the department of General Surgery, Huashan Hospital. His research currently is centered on the mechanism of early metastasis of hepatobiliary and GI malignancies.

Dr. Zheng Wang obtained his M.D. in Cancer Metastasis Institute, Fudan University. He is the chief resident in the department of General Surgery, Huashan Hospital. His research currently is centered on the mechanism of early metastasis of hepatobiliary and GI malignancies. 